\title{
Effects of Necrostatin-1, an Inhibitor of Necroptosis, and its Inactive Analogue Nec-1i on Basal Cardiovascular Function
}

\author{
A. SZOBI ${ }^{1}$, T. RAJTIK ${ }^{1}$, A. ADAMEOVA ${ }^{1}$ \\ ${ }^{1}$ Comenius University in Bratislava, Faculty of Pharmacy, Department of Pharmacology and \\ Toxicology, Bratislava, Slovak Republic
}

Received June 2, 2016

Accepted August 12, 2016

\begin{abstract}
Summary
Inhibition of receptor-interacting serine/threonine-protein kinase 1 (RIP1) by necrostatin-1 (Nec-1) alleviates cardiac injury due to prevention of necroptotic cell death. Its inactive analogue necrostatin-1i (Nec-1i), lacking RIP1 activity, serves as a suitable control. It is unknown if these agents influence the heart function in the absence of damaging stimuli. For this purpose, we measured intraarterial blood pressure (systolic - SBP and diastolic - dBP) and ECG parameters after a bolus administration of $\mathrm{Nec}-1$ and $\mathrm{Nec}-1 \mathrm{i}$ in rats during $30 \mathrm{~min}$. Nec-1, unlike $\mathrm{Nec}-1 \mathrm{i}$, increased SBP and $\mathrm{dBP}$, as well as heart rate reaching the peak at $20 \mathrm{~min}$. The $\mathrm{P}$ wave duration tended to be decreased and the duration of the PR interval was shortened by $\mathrm{Nec}-1$ indicating faster conduction of the impulses through atria to the ventricles. The drugs did not influence the QTc interval duration and no episode of ventricular arrhythmia was observed. In summary, Nec-1 temporarily modulates blood pressure and electrical function of the healthy heart. These effects of Nec-1 are likely due to its off-target action or RIP1 has an important role in the regulation of cardiovascular function independently of its action on the necroptotic pathway.
\end{abstract}

\section{Key words}

Necrostatin-1 • Necrostatin-1i • Necroptosis • Heart

\section{Corresponding author}

A. Adameova, Department of Pharmacology and Toxicology, Faculty of Pharmacy, Comenius University, Odbojarov 10, 83232 Bratislava, Slovak Republic. Fax: +421 250117 100. E-mail: adameova@fpharm.uniba.sk
A recently discovered cell death called necroptosis resembles some morphological features of passive necrosis and is strictly orchestrated by a complex of proteins. Precise mechanisms of necroptosis, mainly those involved in its execution, are not fully understood. However, there is increasing evidence that the activation of signaling involving receptor-interacting serine/threonine-protein kinase 1 and 3 (RIP1, RIP3) and a pseudokinase mixed lineage kinase domain-like protein (MLKL) promotes dysregulation of ion homeostasis leading into membrane oncosis. As a consequence, there is plasma membrane disruption and finally the release of intracellular content into the surrounding environment eliciting the inflammatory response (Zhou and Yuan 2014, Xia et al. 2016). In addition, through disrupted membrane of the dying cells, several immunostimulatory molecules are released to stimulate immune response or activate prosurvival processes promoting wound repair (Zong and Thompson 2006).

Identifying the necroptotic RIP1-RIP3-MLKL pathway has indicated that a blockade of either of these proteins can limit the extent of this type of cell death and thus it can be a promising pharmacological target. For inhibition of RIP1, a group of agents called necrostatins (for instance necrostatin-5, -7 and the most used necrostatin-1) is used. In various models of myocardial injury, the inhibition of RIP1 by necrostatin-1 has been found to reduce cellular injury, prevent undesirable remodeling, and improve function (Adameova et al. 2016, Oerlemans et al. 2012). This agent, chemically methyl-thiohydantoin-tryptophan, also acts as an inhibitor of the potent immunomodulatory enzyme indoleamine 
2,3-dioxygenase, thereby possessing a possible ability to modulate tissue injury independently of RIP1 inhibition. Its inactive variant Nec-1i, (identical to Nec-1 without a methyl group) lacking the RIP1-targeting effects is also predicted to inhibit this enzyme (Takahashi et al. 2012). Thus, immunomodulation produced by both Nec-1 and Nec-1i can influence the extent of myocardial injury and thereby causing misinterpretation of findings in some cases. In addition, it has not been tested if these agents possess additional off-target pharmacodynamic effects modulating the heart function. In this context, we investigated for the first time the effects of a bolus dose of Nec-1 and Nec-1i on blood pressure and basic electrocardiogram parameters in rats during a 30-min long period after intravenous application.

Protocol has been approved by the Ethics Committee of the Faculty of Pharmacy, Comenius University. All described procedures were performed in accordance with the Guide for the care and Use of Laboratory Animals, published by the US National Institutes of Health (NIH publication No 85-23, revised in 1996), preceding an authorization by the Animal Care and Use Committee of Slovak Republic.

Healthy adult male Wistar rats (initial body weight 240-300 g, Dobrá Voda, SAV, SR) were housed in a controlled environment (12:12 light/dark cycle, $23 \pm 1{ }^{\circ} \mathrm{C}$, humidity $55 \pm 5 \%$ ) and given a standard pelleted diet and water ad libitum. After adaptation period, the rats ( $\mathrm{n}=5-7$ per group) were randomized into 3 different groups - control (C), necrostatin-1 perfused (Nec-1) and necrostatin-1i perfused (Nec-1i).

In heparinized $(2,000 \mathrm{IU} / \mathrm{kg})$ and anesthetized rats (isoflurane, $4 \%$ for induction, $1.2-2.0 \%$ for maintenance), 3-lead ECG electrodes connected to a BioAmp (ADInstruments, New Zealand) were inserted subcutaneously and the left carotid artery was cannulated. Carotid catheter filled with heparin-containing saline was connected to a physiological pressure transducer linked with a PowerLab setup (ADInstruments, New Zealand). Animals were left to stabilize for at least $30 \mathrm{~min}$. The right femoral vein was accessed and necrostatin-1 ( $0.8 \mathrm{mg} / \mathrm{kg}$; Nec-1 group), necrostatin-1i $(0.846 \mathrm{mg} / \mathrm{kg}$; equimolar and purity adjusted; Nec-1i group) or vehicle $(20 \% \mathrm{v} / \mathrm{v}$ DMSO in saline; $\mathrm{C}$ group) was applied intravenously as a bolus over the course of around $10 \mathrm{sec}$. ECG parameters and blood pressure were monitored for $30 \mathrm{~min}$.

Data are presented as deltas compared to the stabilization period in the form of mean \pm SEM.
Consecutive 10-min intervals were averaged to obtain the used data points (last $10 \mathrm{~min}$ of stabilization and minutes 0-10, 10-20 and 20-30 post injection). Group differences in variables with normal distribution were tested for by using mixed-model ANOVA followed by Dunnett's test. Statistical analyses were performed with GraphPad Prism version 6.00 for Windows (GraphPad Software, USA). Differences between groups were considered to be significant at $\mathrm{p} \leq 0.05$.

Although Nec-1 and Nec-1i have been widely used to examine necroptosis in myocardial pathologies (Oerlemans et al. 2012, Koudstaal et al. 2015, Dmitriev et al. 2013, Smith et al. 2007, Lim et al. 2007, Miyamae et al. 2014) effects of these agents on basal function of the cardiovascular system have not been investigated so far. In this study conducted in healthy rats, delta sBP recorded at $30 \mathrm{~min}$ after the drug administration did not differ among the groups. However, Nec-1 induced the initial increase of sBP which was observed during the first two 10-min intervals. At the end of the experiment, the values of delta $\mathrm{dBP}$ were similar in all experimental groups. However, there was a gradual increase in delta dBP in the Nec-1 group reaching the significance at 20 min (Fig. 1A, B). These effects of Nec-1, which can be attributed in part to the working myocardium, are consistent with a study of Dmitriev et al. (2013), who have shown a marked initial elevation of systolic intraventricular pressure. In contrast to our findings, diastolic BP after intracoronary Nec-1 application was unchanged (Dmitriev et al. 2013).

The same pattern of changes as observed in BP was observed while measuring heart rate. Indeed, Nec-1, but not Nec-1i, induced an increase in HR reaching the peak at 20 min after the drug administration (Fig. 1C). However, it should be pointed out that in spite of this increase in HR, no ventricular tachycardia nor fibrillation was recorded at any of timepoints of the protocol. Although there were several protocol differences in comparison to ours (the used dose, application into the femoral vein vs. intracoronary application), Dmitriev et al. (2013) have also shown HR values being unchanged at 10 min after Nec-1 administration. On the other hand, as they have monitored heart function only during a short 10-min stabilization period prior to ischemia/reperfusion, it cannot be ruled out that heart rate was also changed at a later timepoint after the drug administration like seen in our hands. 
A)

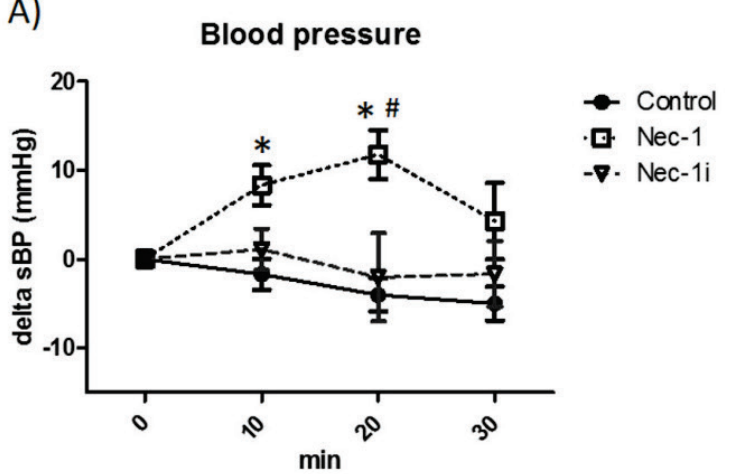

B)

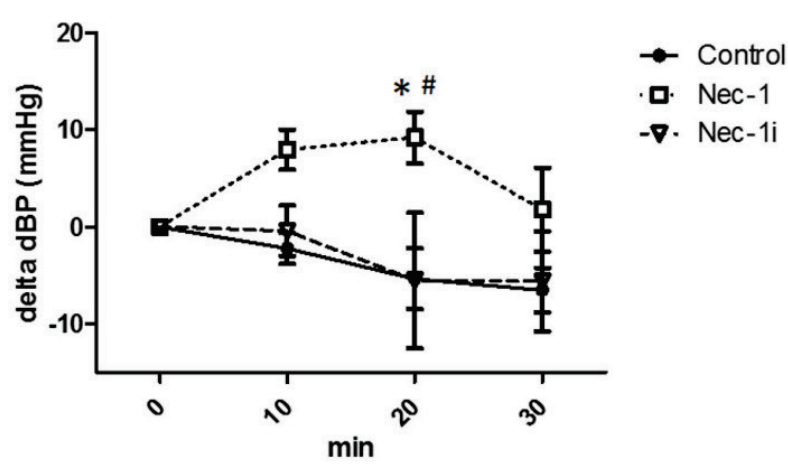

C)

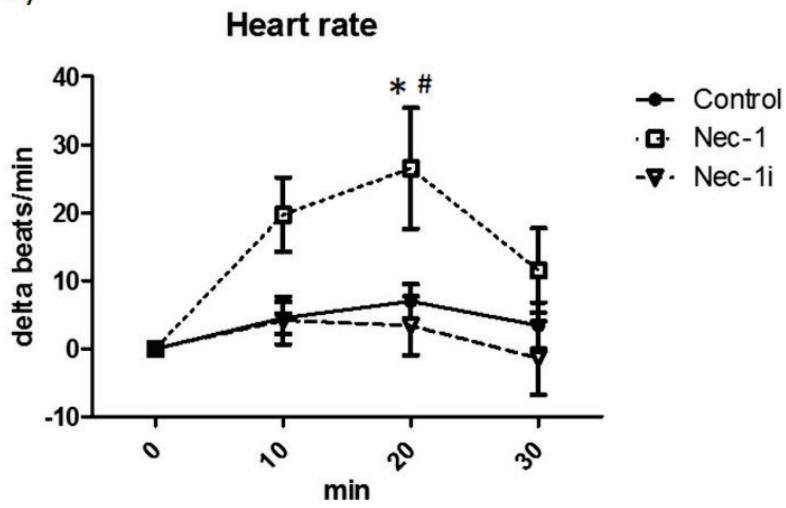

Fig. 1. Effects of a bolus injection of necrostatin-1 (Nec-1) and necrostatin-1i (Nec-1i) on delta systolic (sBP) and diastolic (dBP) blood pressure (A, B) and heart rate (C) during 30 min analyzed in consecutive 10-min intervals. Data are expressed as deltas compared to the stabilization period in the form of mean \pm SEM. $* p \leq 0.05$ vs. Nec- $1 ; \# p \leq 0.05$ vs. C.

Alterations in the duration of certain ECG parameters, both shortening and prolongation, is known to produce electrical instability and the probability of arrhythmias development. After Nec-1 injection, the $\mathrm{P}$ wave duration, an indicator of atrial depolarization, tended to be shortened during the entire 30 -min period. In contrast, Nec-1i did not alter the duration of atrial depolarization. ECG analysis further revealed that the duration of PR interval, referring to atrioventricular conduction velocity to the ventricle, was shorter in the Nec-1 group during 20 min after injection as compared to the group treated with Nec-1i. The QT interval duration, which is precisely monitored because of its association with ventricular tachyarrhythmias (Shah 2010), was unchanged by neither of the drug (not shown). Likewise, the QT interval corrected to heart rate (QTc) did not significantly differ among the groups, albeit a mild nonsignificant prolongation was observed at 10 -min point in the Nec-1 group as compared to control group (Fig. 2A, B).

The data presented in this study indicate that only Nec-1 temporarily modifies basal sBP, dBP, HR and some ECG parameters and that Nec-1i-treated group does not significantly differ from the control group treated with a vehicle. Thus, it can be hypothesized that Nec-1 possess off-target RIP1-independent activity or that RIP1 has additional effects directly regulating cardiovascular function. As Nec-1i, extremely similar to Nec-1 with no ability to inhibit RIP1, has not influenced hemodynamic nor electrical activity of the heart, the latter option rather than the former one explains these findings. Indeed, changes observed in this study might suggest the possibility of Nec-1 being capable of modulating the autonomic nervous system, likely the sympathetic arm which directly regulates both heart function and vascular tone. A critical role of RIP1 has been highlighted in a study showing that RIP1 knockout causes postnatal lethality (Kelliher et al. 1998), thereby indicating its prerequisite for vital function of the organism. In line, a recent study has suggested that RIP3, being a downstream target of RIP1, activates CaMKII (Zhang et al. 2016) which is known to phosphorylate and thus regulate excitation-contraction coupling of the heart (Luo et al. 2013). The link between CaMKII, RIPs, necroptotic cell death and the force of contractile function has been proposed for the first time by our group (Szobi et al. 2014). In fact, we have found that inhibition of CaMKII normalizes the upregulation of RIP1 levels and that these changes are linked with the improved contraction cycle in $\mathrm{I} / \mathrm{R}$ heart.

From the foregoing discussion it is plausible that RIP1, besides its participation in signaling promoting cell death, is implicated in pathways regulating both pacemarker cells and nonpacemaker cardiomyocytes responsible for regulation of blood pressure. While the former action of RIP1 is mainly relevant in the presence of pathological impulse, the latter might be continuously active and independent of particular heart conditions. 
A

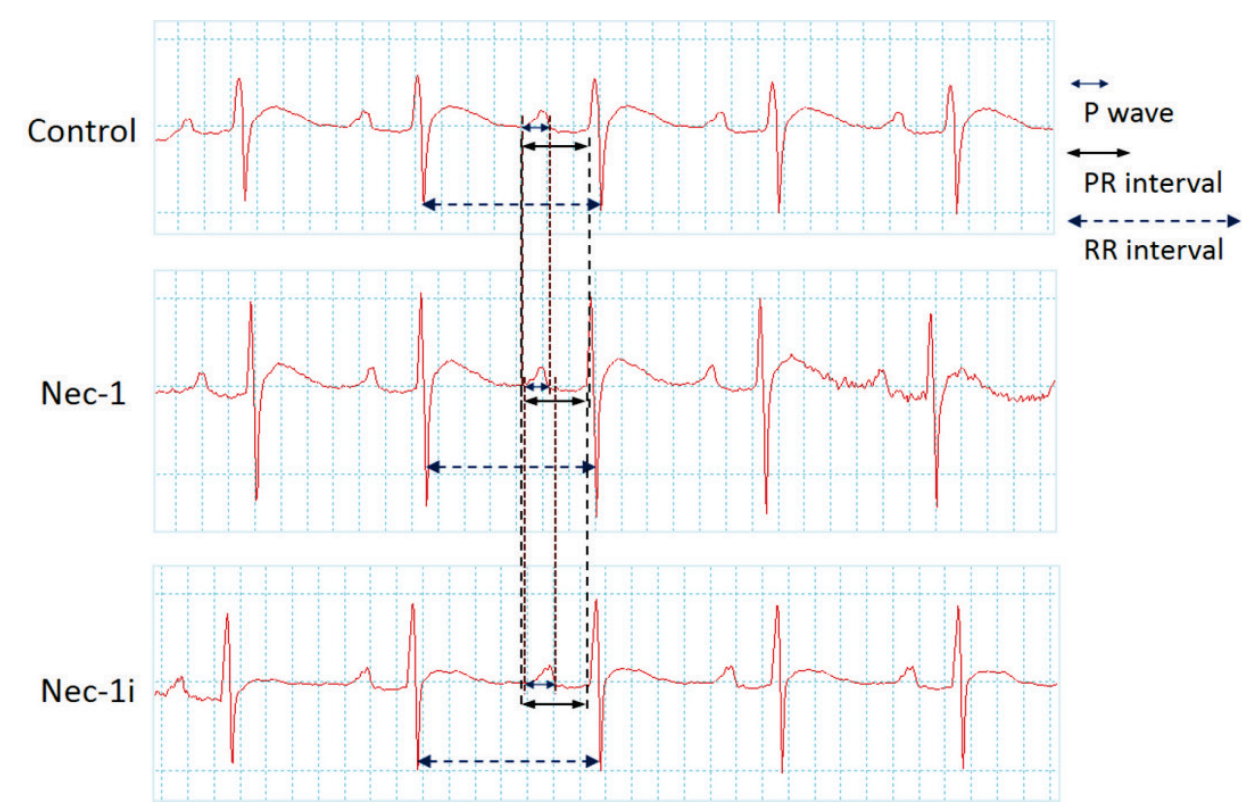

B

P wave

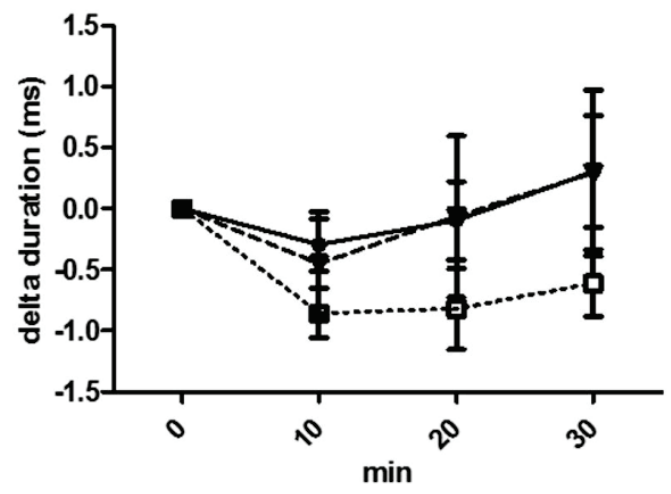

RR duration

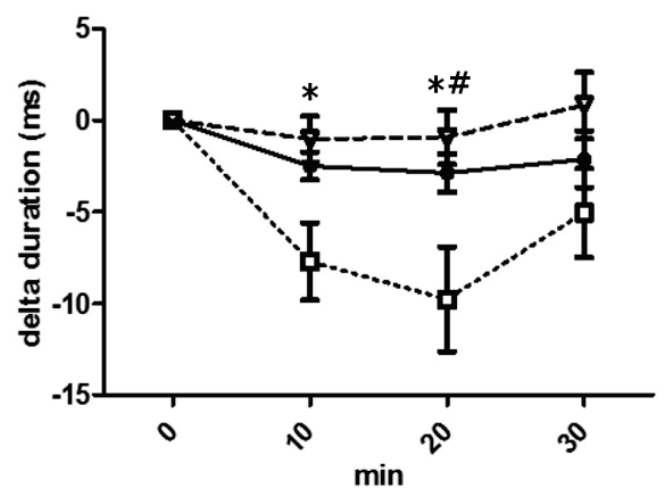

PR interval

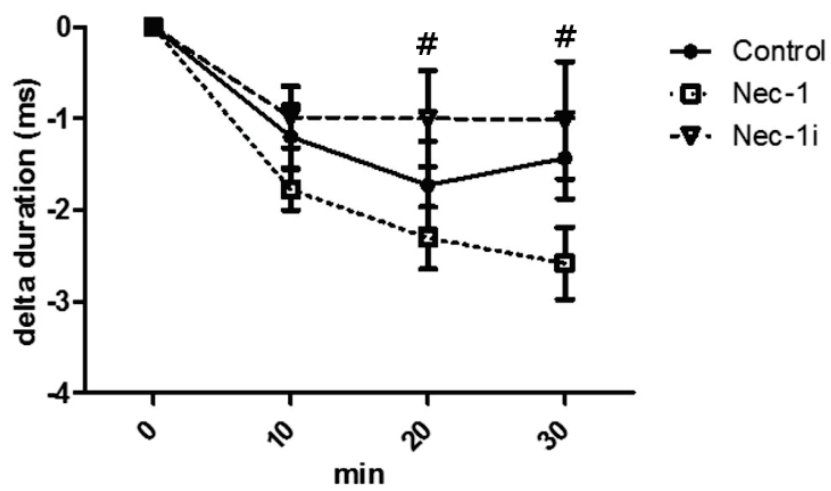

QTc interval

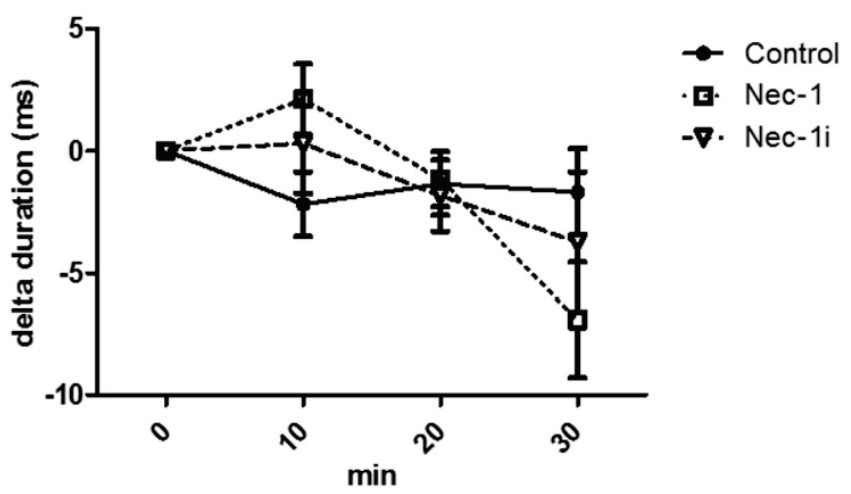

Fig. 2. Modulation of electrical activity of the heart due to administration of necrostatin-1 (Nec-1) and necrostatin- $1 \mathrm{i}$ (Nec-1i). A) Representative ECG tracings of a control (C), Nec-1-treated (Nec-1) and Nec-1i-treated rat indicating the P wave, PR interval and RR abnormalities. B) Changes in the duration of the P wave, PR interval, and QT interval corrected to heart rate (QTC). Data are expressed as deltas compared to the stabilization period in the form of mean \pm SEM. $* p \leq 0.05$ vs. Nec- $1, \# p \leq 0.05$ vs. C. 


\section{Conflict of Interest}

There is no conflict of interest.

\section{Acknowledgements}

This study was supported by VEGA 1-0271-16, Slovak Society of Cardiology and APVV-15-607. The authors thank Mrs. Hassova for her excellent technical assistance.

\section{References}

ADAMEOVA A, GONCALVESOVA E, SZOBI A, DHALLA NS: Necroptotic cell death in failing heart: relevance and proposed mechanisms. Heart Fail Rev 21: 213-221, 2016.

DMITRIEV YV, MINASIAN SM, DEMCHENKO EA, GALAGUDZA MM: Study of cardioprotective effects of necroptosis inhibitors on isolated rat heart subjected to global ischemia-reperfusion. Bull Exp Biol Med 155: 245-248, 2013.

KELLIHER MA, GRIMM S, ISHIDA Y, KUO F, STANGER BZ, LEDER P: The death domain kinase RIP mediates the TNF-induced NF-kappaB signal. Immunity 8: 297-303, 1998.

KOSHINUMA S, MIYAMAE M, KANEDA K, KOTANI J, FIGUEREDO VM: Combination of necroptosis and apoptosis inhibition enhances cardioprotection against myocardial ischemia-reperfusion injury. $J$ Anesth $\mathbf{2 8}$ : 235-241, 2014.

KOUDSTAAL S, OERLEMANS MI, VAN DER SPOEL TI, JANSSEN AW, HOEFER IE, DOEVENDANS PA, SLUIJTER JP, CHAMULEAU SA: Necrostatin-1 alleviates reperfusion injury following acute myocardial infarction in pigs. Eur J Clin Invest 45: 150-159, 2015.

LIM SY, DAVIDSON SM, MOCANU MM, YELLON DM, SMITH CC: The cardioprotective effect of necrostatin requires the cyclophilin-D component of the mitochondrial permeability transition pore. Cardiovasc Drugs Ther 21: 467-469, 2007.

LUO M, ANDERSON ME: Mechanisms of altered $\mathrm{Ca}^{2+}$ handling in heart failure. Circ Res 113: 690-708, 2013.

OERLEMANS MI, LIU J, ARSLAN F, DEN OUDEN K, VAN MIDDELAAR BJ, DOEVENDANS PA, SLUIJTER JP: Inhibition of RIP1-dependent necrosis prevents adverse cardiac remodeling after myocardial ischemiareperfusion in vivo. Basic Res Cardiol 107: 270, 2012.

SMITH CC, DAVIDSON SM, LIM SY, SIMPKIN JC, HOTHERSALL JS, YELLON DM: Necrostatin: a potentially novel cardioprotective agent? Cardiovasc Drugs Ther 21: 227-233, 2007.

SHAH RR: Drug-induced QT interval shortening: potential harbinger of proarrhythmia and regulatory perspectives. $\mathrm{Br}$ J Pharmacol 159: 58-69, 2010.

SZOBI A, RAJTIK T, CARNICKA S, RAVINGEROVA T, ADAMEOVA A: Mitigation of postischemic cardiac contractile dysfunction by CaMKII inhibition: effects on programmed necrotic and apoptotic cell death. $\mathrm{Mol}$ Cell Biochem 388: 269-276, 2014.

TAKAHASHI N, DUPREZ L, GROOTJANS S, CAUWELS A, NERINCKX W, DUHADAWAY JB, GOOSSENS V, ROELANDT R, VAN HAUWERMEIREN $F$, LIBERT C, DECLERCQ $\mathrm{W}$, CALLEWAERT $\mathrm{N}$, PRENDERGAST GC, DEGTEREV A, YUAN J, VANDENABEELE P: Necrostatin-1 analogues: critical issues on the specificity, activity and in vivo use in experimental disease models. Cell Death Dis 3: e437, 2012.

XIA B, FANG S, CHEN X, HU H, CHEN P, WANG H, GAO Z: MLKL forms cation channels. Cell Res 26: 517-528, 2016.

ZHANG T, ZHANG Y, CUI M, JIN L, WANG Y, LV F, LIU Y, ZHENG W, SHANG H, ZHANG J, ZHANG M, WU H, GUO J, ZHANG X, HU X, CAO CM, XIAO RP: CaMKII is a RIP3 substrate mediating ischemia- and oxidative stress-induced myocardial necroptosis. Nat Med 22: 175-182, 2016.

ZHOU W, YUAN J: Necroptosis in health and diseases. Semin Cell Dev Biol 35: 14-23, 2014.

ZONG WX, THMPSON CB: Necrotic death as a cell fate. Genes Dev 20: 1-15, 2006. 